\title{
How child mental health training is conceptualized in four low- and middle-income countries
}

\author{
Panos Vostanis $^{1^{*} \mathbb{D} \text {, Seyda Eruyar }}{ }^{2}$, Sadiyya Haffejee ${ }^{3}$ and Michelle O'Reilly ${ }^{1}$
}

\author{
${ }^{*}$ Correspondence: \\ pv11@le.ac.uk \\ ${ }^{1}$ University of Leicester, \\ Leicester, UK \\ Full list of author information \\ is available at the end of the \\ article
}

\begin{abstract}
The objective was to establish how stakeholders in low- and middle-income countries (LMIC) conceptualize child mental health impact. Semi-structured interviews were conducted with 18 stakeholders from disadvantaged urban communities in Kenya, South Africa, Turkey and Brazil. Participants represented education, welfare and health care agencies; as well as community and religious groups. Data were analysed through a thematic approach and three related themes were identified. Impact was defined as a process of change, with child-centred outcomes and measures that were broader than mental health symptoms improvement. Beneficiaries were identified at child, family, community and service level. Participants wished to see strategies that ensured sustainability of impact, namely child mental health policy, stakeholder engagement, interdisciplinary working, and capacity-building for all stakeholder groups. Child mental health service transformation in LMIC, especially in areas of deprivation, needs to build on existing resources and strengths by co-producing psychosocial outcomes with a range of professional and community stakeholders. Impact on children's mental wellbeing can be achieved through a co-ordinated strategy that involves designated policy, capacity-building, and interdisciplinary networks with meaningful community involvement.
\end{abstract}

Keywords: Child, Mental health, Psychosocial, Services, Policy, Training, Impact, Stakeholders, Low- and middle-income countries

\section{Introduction}

Children's mental wellbeing is increasingly becoming a global concern (UN, 2014). It is established that one in ten children and young people under 18 years have mental health problems that would benefit from assessment and intervention (Skokauskas et al., 2019). These prevalence rates rise to at least double in areas of disadvantage, because of associated risk factors such as poverty, family and community violence (Hussain et al., 2012), and to $40-50 \%$ among groups exposed to multiple and recurrent traumatic events such as refugee, street and orphaned children (Ali et al., 2020; Eruyar et al., 2018; Myburgh et al., 2015). Vulnerabilities are more pronounced in low- and middle-income countries (LMIC), especially in deprived communities like urban informal settlements (Bele et al., 
2013). In these contexts, children are more susceptible to maltreatment, illegal labour and sexual exploitation (Pandian \& Lakshmana, 2017; Reza \& Bromfield, 2019). Lack of early intervention and supporting systems can affect children's later psychosocial outcomes along different life domains (Frankish et al., 2018; Patel et al., 2018).

Despite this evidenced high level of mental health need, access to appropriate mental health and related services remains limited in LMIC (Academy of Medical Sciences, 2018; Pedersen et al., 2019). Help-seeking barriers further hinder the problem and include stigma of mental illness, parental engagement and culturally appropriate interventions (Getanda et al., 2017). These barriers are compounded by lack of designated policy and limited resources in terms of skilled professionals, infrastructure and available services (Patel et al., 2018). Reasons for these limited resources in LMIC can be attributed to poverty and competing demands on the fiscal budget, broader systemic challenges (within welfare, education and healthcare), shortages of trained staff, and lack of prioritization of psychosocial care (Patel \& Rahman, 2015; UNHSP, 2017).

Culture is a key factor in a child's macro-system, therefore, it is important to consider theories and evidence on its influence on child development, onset and expression of mental health problems, parenting practices, attitudes to supports and services, and design of interventions (Reardon et al., 2017). Of those theories, this study was particularly informed by the impact of culture on help-seeking. The conceptualization of mental health and illness varies across and within sociocultural contexts, for example between urban and rural areas (Tamburrino et al., 2020). Certain presentations, mainly of internalizing nature like as depression and anxiety, may not be viewed as mental health problems, whilst the causes of other conditions may be attributed to a range of causes (Moller et al., 2016). Cultural perceptions and beliefs can thus lead to both personal and public stigma, which consequently act as barriers to seeking help and engaging with services (Schnyder et al., 2017).

Additional constraints in child mental health service provision include the lack of child-centred guidelines, as available policy briefs are largely adult-focused, and the absence of stakeholder involvement (WHO, 2016). As children's mental wellbeing is relevant to a range of agencies, stakeholders include education (schools, special educational needs and educational psychology), health care (primary and hospital-based), welfare (child protection, family support, community and residential care settings), and non-governmental organizations (NGOs); as well as service user (children, young people and families), community and religious groups (Vostanis, 2017). Capturing these voices, and wealth of local knowledge and expertise are essential for service transformation, i.e. for the re-orientation and improvement of service delivery systems, with the service user in the centre. Systemic changes, for example, should aim at services becoming more relevant, equitable in terms of access, responsive, person-centred, evidence-based and more efficient (Adams et al., 2009). Users should be actively involved in all stages of service transformation, i.e. planning, scaling-up and monitoring (Patel et al., 2018). A central concept in service transformation is the co-dfinition of what constitutes acceptable and contextualized service impact (ESRC, 2019).

To date, there is relative consensus on what constitutes impact of policy, services and interventions for major health conditions such as obesity and diabetes (Nianogo \& Arah, 2018), including in LMIC populations (Bawah et al., 2019; Kontis et al., 2019). There is 
less stakeholder-led evidence, however, on what should constitute desirable impact of child mental health interventions and services. Several researchers, for example, argue that impact should be broader than improvement in mental health symptoms, and include outcomes such as optimal family functioning, quality of life, resilience, and school attainment (Boylan, 2018; Wolpert et al., 2017). Existing evidence on definitions of impact and associated outcomes is predominantly based on community-based (Garcia-Carrion et al., 2019; O'Connor et al., 2017) and specialist children's interventions and services in high-income countries (Lavik et al., 2018; Vostanis et al., 2015). Taking into consideration the previously discussed variation in beliefs, needs and systems across LMIC (Tamburrino et al., 2020), establishing the perspectives of stakeholders from different disciplines in LMIC is important in informing policy and service transformation. The importance of co-producing solutions with stakeholders in LMIC is increasingly acknowledged. In recent years, a range of policy makers, professionals and community leads have been involved in scoping studies (Vostanis et al., 2018) or protocols for the implementation of interventions (Kumar et al., 2020). Stakeholder groups, however, often do not involve children or youth, and are not embedded in service design, delivery and monitoring. This policy and research gap informed the rationale for this study.

\section{Methods}

The aim of this study was to establish how stakeholders in four LMIC conceptualized impact on service delivery, following child psychosocial service transformation training. The following research questions were addressed:

1. At what levels (community, practice, service, policy) did interdisciplinary stakeholders perceive impact?

2. To what extent were perspectives similar or different across the four groups?

\section{Context and participants}

Participants were recruited from a larger interdisciplinary sample of professionals, managers and community or religious leaders $(n=98)$, who had attended child psychosocial service transformation training in four LMIC cities (Nakuru, Kenya; Istanbul, Turkey; Rio de Janeiro, Brazil; and Johannesburg, South Africa) 6 months earlier. This training, its underpinning framework and pilot evaluation are described in detail elsewhere (Vostanis et al., 2019).

In summary, in each country the target group consisted of children and youth living in a disadvantaged area (or informal settlements, often referred to as slums or favelas) was identified. A host NGO worked with the research team to organize the training based on the stepped service framework, which acted as a template. This framework was contextualized and co-adapted to local circumstances and needs between the host NGO and the first author, who subsequently jointly facilitated the child psychosocial transformation workshop. Child mental health problems were defined as a "recognisable set of symptoms or behaviours associated in most cases with considerable distress and substantial interference with personal functions" (Green et al., 2005, p.8). However, as participants worked in contexts of disadvantage, where children were faced with multiple 
and inter-linked needs, these can be better understood under a broad 'psychosocial' definition, which refers to the dynamic inter-connection between psychological (internal, emotional and thought processes, feelings and reactions) and social (relationships, family and community networks, social values and cultural practices) functions (InterAgency Standing Committee, 2007).

All agencies (education, welfare, health and non-statutory) and community groups in contact with children were invited to the workshop. Participants were initially asked to map children's needs, existing resources and supports, service gaps, barriers and priorities in their area. They were subsequently presented with the contextualized framework of six domains: child safety, caregiver support, enhancing resilience through schools and communities, upskilling frontline professionals and community volunteers, counselling, and accessing child mental health services. Participants were asked to co-devise realistic and achievable action plans on each domain for the following 6 months. Although the specific action plans related to local circumstances, we analysed the overall dataset and established the emerging strategies that underpinned the recommended action plans.

For the purpose of the study reported in this paper, selection criteria included five participants from each country; who had attended the training 6 months earlier; worked in a capacity that could locally influence impact; and represented the education, welfare and health and community sectors as far as possible at each site. Three rather than the intended five stakeholders were reached in Brazil, because of eruption of violence in the selected area of favelas during the period of recruitment. The sample thus comprised 18 participants. Their professional disciplines and agency roles within each country are presented in Table 1. Participants worked with children and youth aged 0-18 years. Their training was broadly similar in terms of undergraduate first degree qualifications and duration of training for teachers, psychologists and medics. This may indicate a degree of self-selection in terms of socioeconomic status.

This sample size was sufficient to secure sampling adequacy and reach coding saturation (see Hennink et al., 2017) across countries, but not within countries. This was in

Table 1 Participants professional roles $(n=18)$

\begin{tabular}{ll}
\hline Country/site & Professional/agency role \\
\hline Brazil & Co-ordinator of social project \\
& Co-ordinator of school re-integration project \\
Kenya & Reading mediator \\
& Pastor \\
& Co-ordinator of community faith-based project \\
& Counselling teacher \\
& Teacher and mentor \\
& Teacher \\
South Africa & Life coach \\
& Care manager (residential and community) \\
& Social worker \\
& Child and youth worker \\
& Psychologist \\
Turkey & Residential care worker \\
& Family physician \\
& Co-ordinator for education of orphan children \\
& Psychologist and community project co-ordinator \\
& Manager of education project for refugee children \\
\hline
\end{tabular}


part due to the professional status of the participants having some degree of educational similarity, and in part due to the combined inductive and deductive processes (see analysis) providing a degree of structure to the process, which meant less interviews were needed (Braun \& Clarke, 2019; Hennink et al., 2017). Furthermore, the groups were previously seldom heard, and their narratives were rich in detail and meaning, thus ensuring that the coding captured the core issues at stake from voices rarely reflected in the literature, without placing additional burden on these groups by sampling further than necessary.

The study was approved by the Research Ethics Committee of the University of Leicester in the UK. Data were collected through semi-structured interviews, which took place in the native language. All information included in the interview transcripts was anonymized and participants were given a code number. Their broad professional backgrounds were maintained, in order to give a context of agencies and professional roles involved with the children. Participants were reassured that no quotes which could, direct or indirectly, identify them would be included in the dissemination. Audio recordings were transcribed by the researcher who had conducted the interviews in each country, with the exception of South Africa where an independent professional transcriber was used. The same transcriber (one in each country) translated the interviews into English, following which all transcripts were pooled into one dataset for the coding.

\section{Data analysis}

Thematic analysis was the adopted framework in identifying, analysing and reporting patterns (themes) within the data (Braun \& Clarke, 2006). This was congruent with the open nature of the research questions and the goal of identifying common issues at stake across country professional groups. Both inductive and deductive methods were utilized, as the researchers had not adopted a pre-existing coding frame while searching for new concepts in the dataset, yet identified the themes related to the research questions and the wider literature in conjunction with the data. This combination of deductive and inductive, structured and unstructured approach to conceptualization and identification of categories within the data, as underpinned by the broader literature and goals of the research project, is congruent with a 'codebook' approach to thematic analysis (Clarke \& Braun, 2018). The codebook approach was thus the method of choice, as it uses a combination of deductive and inductive analysis, whereby the coding is participant-driven, but will utilize the structure of the interview guide and wider evidence, where relevant, to facilitate the creation and organization of the codes. This deductive aspect can also help with the labelling of certain codes, where there is uncertainty.

Clarke and Braun (2018) report that the 'codebook' approach is a thematic analysis that allows for a priori literature to facilitate the identification of coding to be combined with participants' narrative, and is more structured, than the reflexive approach (see also Braun \& Clarke, 2019). Braun and Clarke (2019) argued that the codebook approach to thematic analysis means that most codes are developed from the first few interviews, and this creates the coding framework for the rest, meaning that saturation of data is reached sooner. This method thus has the benefit of creating a coding frame and determining conceptual categories that are driven by the data, participants, and wider research knowledge. The NVivo 12 programme was used to facilitate coding. Two coders (SH and 
PV) were involved who, through consultation and mapping the two frames against one another, identified disagreement or gaps, and merged them together for a final agreement. A third independent team member (MOR) helped resolve any discrepancies. This process thus assured cohesion across coding and results in coder agreement.

\section{Results}

Thematic analysis resulted in the identification of six overarching themes. In the present paper, the three themes which address the research questions are reported (Table 2). The remaining three themes were related to the implementation of the training, identified challenges and future training strategies.

\section{Theme 1: conceptualization and measurement of impact}

Subjective and objective measures of impact are both important, especially in the field of child mental wellbeing. Responses coded within this theme represented participants' own conceptualization of 'impact' and the indicators they used to evaluate this. Impact was usually related to stakeholders' agency and professional role. Twelve participants described the impact and provided a rich definition, six of whom defined it as 'change' or 'transformation'. This change was given mostly positive connotations, whilst some participants acknowledged that this could also be bidirectional in potentially resulting in deterioration of a child's problems, if professionals were not adequately skilled and/or interventions not appropriate. Metaphors of 'pictures' were used by two participants as capturing change.

"It's to see, to see something different from before to now. Just like photographers are very good in taking photographs of a picture of, of something old, and a new picture, and they show the change. I think to me change is when you see the transformation."

Community Project Co-ordinator, Kenya

Participants' suggestions on how to measure outcomes of change reflected the literature on the complexity of child mental health needs, their association with educational and welfare correlates, and their different expression in different contexts (Arora et al., 2016). Improvement in mental health symptoms was the most commonly reported indicator. This usually related to externalizing (conduct) symptoms such as

Table 2 Emerging themes and sub-themes

\begin{tabular}{ll}
\hline Theme & Sub-theme \\
\hline Conceptualization and measurement of impact & Change and transformation \\
& Child's wellbeing \\
& Academic attainment \\
& Pre- and post-assessment \\
& Observation of behaviour \\
Beneficiaries of impact & Children \\
& Parents \\
Sustainability of impact & Professionals \\
& Services \\
& Policy \\
& Raising awareness \\
& Stakeholder engagement \\
& Capacity-building \\
\hline
\end{tabular}


aggression, with some examples on internalizing (emotional) functions like 'not talking' being reported. Broader outcomes largely referred to academic attainment and social interactions, for example through play for younger children. In response to a question on how and when to measure change, responses were aligned to child mental health practice guidelines, by involving different informants (child, parent and teacher), pre- and post-intervention measurement, and arranging medium-term follow-up to ensure that benefits are sustained (De Los Rayes et al., 2015).

"But I would like a little page of where the child's at, and where we see the child going, and how we're gonna help them step-by-step. So, even if we can measure that over six months."

Life coach, South Africa

\section{Theme 2: beneficiaries of impact}

Achieving impact was contextualized in terms of those who reaped the benefits. Four beneficiary groups were thus identified at child, parent, community and service level, which reflect the dynamically linked socioecological systems (Bronfenbrenner, 1979). Different implications were consequently described for each beneficiary group.

Children with mental health problems should benefit along different domains of psychosocial functioning that can be impaired through ill mental health. Participants delved more on the process of achieving such impact, and also what this meant for the child's everyday life. Examples were provided for the behavioural and emotional domains, and their ability to regain control and function autonomously. For children who experienced traumatic events, the goal could be to feel comfortable to share difficult experiences and emotions. Psychosocial improvement was linked to academic attainment, which should also include life and employment skills.

"I think we're seeing results now, children are more open to talk about and express their feelings; because, I think it's being pushed a long way, and I think it is creating more safe space to do."

Community Project Co-ordinator, Turkey

A pre-requisite for achieving child-focused psychosocial outcomes was ensuring the child's physical and emotional safety, which is consistent with the hierarchy of first meeting their basic needs (Maslow, 1943). In addition to implementing child protection procedures, developing a trusting relationship with children was essential in making them feel sufficiently safe to benefit from a therapeutic intervention. The importance of building trust was especially emphasized by South African stakeholders predominantly working in care settings with children who had suffered maltreatment, were therefore more likely to suffer from disrupted attachment relationships (Dalgaard et al., 2016).

"...the relationship between me and the young people that I've engaged with so far, it has strengthened the relationship. So, it's no longer when I talk to them, now there's a relationship.

Care manager, South Africa 
Improving parenting skills and child-parent relationships was a commonly reported outcome, which would also equip children to generate secure relationships and positive outlooks in their school and social environments. The first step was for professionals to engage parents, who might have other priorities such as working long hours out of the home, and/or not being able to recognize their role in the development and maintenance of children's mental health problems. Once they were engaged, strategies aimed at supporting parents to observe, recognize and link parenting strategies with children's mental wellbeing.

"Where the child feels like I'm a child, I'm no longer in that you know, whatever, you know toxic, you know, environment, where the auntie or the mom who shout." Care worker, South Africa

As children were often exposed to similar risk factors such as violence and aggression in different environments (home, school and community), hence variably exhibited mental health problems in these settings, it was considered important to tackle these vulnerabilities holistically. Regular communication and consistent approaches between parents, teachers and other professionals were crucial in achieving change.

"The parents had already ignored him because he was a non-performer, but the parent was not aware that the child had a mental health problem. So, when we discussed with the parent, we came together with the other teachers giving the motivation to the child, positive enforcement... finally the child performed very well."

Counselling teacher, Kenya

Participants acknowledged various indicators and processes of impact on themselves as professionals, in enabling improvement among children and parents. Interestingly, they believed that raising their own awareness was important, but this was also a pre-requisite to acquiring new knowledge and skills. Empathizing with children's and parents' needs and challenges was essential in engaging them and instigating change. Participants reported that changes, not only to their working practices, but also to their ideas, beliefs and motivations for working with children, were also important. Stakeholders reflected that such a learning process required case-based training to enable them to practice within their roles, and this should be complemented by supervision and 'booster' workshops.

"Actually what I learned...you need to go to their level, you need to understand them, and then from there you discuss in a fairly manner; because, if you try to be harsh, even as mother or as parent, even you try to be very harsh, the child will not even open up.

Teacher, Kenya

Mirroring children's often complex needs by adopting a holistic approach was perceived as especially important in involving key adults from the child's wider environment and planning multi-modal interventions. This was perceived as a strength of interdisciplinary training and practice forums (Institute of Medicine, 2015). Nevertheless, participants acknowledged that they had been mostly working in silo. 
Information on other professional and agency roles, therefore, changed their perceptions and enhanced knowledge as to resources that could be accessed to overcome constraints. The transition through knowledge, awareness and motivation of professional identity was considered helpful.

"The child lives together with groups that embody themselves. So, we have to try to reach, if not everyone, at least as many people as possible. It is necessary to draw strategies for this child's wellbeing and for those around them."

Reading Mediator, Brazil

"I realized that every child can be healed in terms of their mental health, when teachers, parents and NGOs collaborate well, as that pyramid in the training suggests."

Education Co-ordinator, Turkey

Although the specific context of service impact appeared to vary across four sites, and to be influenced by sociocultural factors, local systems and target client groups, certain sub-themes emerged that transgressed all countries. For example, and consistent with the previous aspiration of improving practice, participants identified interdisciplinary working as a key indicator of service impact. This was considered particularly important in their LMIC context of facing overwhelming health and social needs, working in isolation, and having limited or no access to specialist child mental health services.

"In the psychosocial pyramid that you have, the most severe cases are at the top, we're making sure some of those cases are followed-up, so each case have their own casework, which is something new to the programme".

Community Project Co-ordinator, Turkey

Although the research sample and dataset did not enable the exploration of crosscultural or cross-systems similarities and differences, middle-income countries such as Turkey and Brazil with relatively more established policies and mental health services came across as being more ready to extend existing networks, a concept which appeared more narrow and less developed in low-income countries like Kenya. Stakeholders from this country mainly instigated collaborations within their organization, as well as with individual actors on the child's closer horizon such as parents, teachers and other professionals. In both cases, successful networks relied on initiating and fostering relationships with other agencies on the ground.

"In our team, it was a leap, because now the educators are more confident in their social network and with the scope of possibilities within the team. They are positive about the partnership with other teams, which strengthened all project actions along the second semester." Social Project Co-ordinator, Brazil

\section{Theme 3: sustainability of impact}

In countries with low-level resources it is essential that support is sustainable, and that the effects of capacity-building, interventions and changes in approach are managed 
longer-term. Responses coded within this theme identified participants' ideas on how impact could be enhanced and sustained in the future. Despite the parity of esteem imbued in western rhetoric and a concern of the World Health Organization, in practice limited funding, lack of awareness, few research priorities, and competing demands mean that mental health is not yet on an equal footing to physical health (Millard \& Wessely, 2014; Morton \& O’Reilly, 2019). Prioritizing children's mental wellbeing by policy was thus an overarching recommendation.

"I think that, from a policy point of view, if we could make that almost as important as physical needs and physical care, it may be able to make an impact, a longlasting impact. But because mental health is usually put on the back foot, we're not worrying about the whole person; we're only worrying about the physical part of the person."

Psychologist, South Africa

Policy implementation should start with mental health awareness campaigns within the family, school and community. Psychoeducation should include community volunteers (paraprofessionals) and practitioners, and should constitute the foundation for mental health training. Networks and community groups working in partnership with government departments and services would ensure continuity. The specific role of religious and spiritual leads was also emphasized.

"I was thinking of the peers at the location, when they go to the location. They also look at those, our children, as weird. I think, if we can have some kind of education in schools, so also, they are, the peers at schools, they understand."

Care worker, South Africa

Several recommendations were made on the importance of capacity-building in sustaining impact for children and families. Training should be adapted to the different needs of community volunteers, universal (frontline) and specialist professionals. Programmes should be systematic and continuous, rather than ad hoc. Some participants advocated for targeted training in selecting representatives from different groups or settings, who could then cascade knowledge internally as child mental wellbeing champions. This could be an effective use of resources.

"I think specifically focused trainings, even though this is focused, if you could have it like more then with a specific set of people. So, instead of trying to train as many people as possible, you pick a group and you start with them."

Psychologist, South Africa

\section{Discussion}

This study established the perspectives of stakeholders from different disciplines in four LMIC on what constitutes impact of service delivery, and how this could be achieved to improve children's mental health needs in resource-constrained communities. These perspectives are central to service transformation, especially in the absence of sufficient specialist provision. In summary, stakeholders adopted a holistic approach in defining desired outcomes, process of implementation and interventions. This approach 
was reflected in their identification of dynamically linked beneficiary groups, and the required interdisciplinary working and multi-modal interventions to address the needs of children and families. Designated policy, awareness campaigns and a capacity-building strategy would ensure sustainability of impact.

These findings can be viewed within the existing evidence and policy in relation to children's mental health needs and provision in LMIC (Patel et al., 2018; WHO, 2016). Overall, despite a considerable extent of stigma of mental illness, stakeholders embraced mental health and wellbeing, and provided rather complex definitions of how evolving services should make a difference in several domains of children's and families' lives. Their conceptualization took into consideration current resources and how to maximize their strengths in a stepped process, rather than solely relying on new or external investment. Indeed, various novel interventions have been described and evaluated in LMIC, especially in post-conflict settings and areas of extreme deprivation, which build on such strengths, for example by mobilizing community volunteers (Jordans et al., 2011; Purgato et al., 2014).

Stakeholders represented a range of agencies that largely provide psychosocial support in LMIC such as schools, welfare and primary healthcare settings (Patel \& Rahman, 2015). Proposed outcomes thus reflected children's functioning, quality of life and school attainment, rather than only changes in mental health symptoms (Wolpert et al., 2017). A key finding and arising implication was that impact should be achieved at different and inter-linked levels, i.e. child, family, community and service; while attributing responsibility to different drivers. This is consistent with the socioecological systems framework (Bronfenbrenner, 1979) and the stepped service approach in other areas of public care (UNHCR, 2013). These frameworks are especially pertinent in informing service planning and transformation in LMIC, where community resources such as resident forums, faith groups, schools and NGO already have a prominent role in psychosocial interventions (Vostanis, 2017).

Despite differences between the participating sites, stakeholders demonstrated clear and rather consistent concepts of the impact they wished to achieve, whilst setting a realistic process of change. This change will obviously depend on complex and interconnected political, cultural and service transformation. For this reason, policy and future research should clearly define, implement and evaluate stages in this process, and for different beneficiary groups (service user, practitioner, service and policy). A range of implementation science approaches (Nilsen, 2015) such as realist evaluation (Pawson \& Tilley, 1997) are useful in capturing and understanding the process or mechanisms through which multiple outcomes are achieved for certain groups and in certain contexts $(\mathrm{CMO}=$ context-mechanism-outcome).

For impact to be sustained, strategies should also be multi-layered and connected. These should include the development of designated mental health policy; integration of mental health into existing child protection, education and physical health policy; establishment of local interdisciplinary networks to implement these policies; building on existing community resources; and capacity-building, including the establishment of a pool of future trainers (Vostanis, 2019). Training initiatives should address different roles in order to upskill community volunteers, universal and specialist professionals (Patel et al., 2018). As previous evidence indicates (e.g. Jalal et al., 2019), capacity-building 
should be planned in conjunction with mental health awareness for the general population and different service provider groups, if acquired intervention skills are to be implemented efficiently by engaging communities.

These impact-promoting strategies were defined in an interdisciplinary context by the stakeholders, despite acknowledging that they previously worked in silo. He et al. (2015) found that such collaboration can lead to better recognition of child mental health problems, thus more cost-effective referrals. In a systematic review that included 33 studies, Cooper et al. (2016) identified joint training as a key facilitator of interdisciplinary collaboration across several countries, a principle which has been endorsed by the World Health Organization (WHO, 2010). Interdisciplinary training in LMIC may potentially have an even higher impact in the absence of well-developed specialist services. Schools and community centres can play a prominent role in hosting interdisciplinary hubs.

The findings should be viewed within the limitations of the study design. Notably, children and parents were not included. These are key stakeholders to help service developers understand and overcome help-seeking barriers such as stigma. The sample was not representative of all agencies operating in the participating countries and communities. It may have carried bias by including more motivated stakeholders and agencies that had already participated in service transformation training. Subjective perspectives should be complemented by measurement of impact through service records, changes in children's functioning, user satisfaction, and staff knowledge. Although it would have been desirable to establish contextual differences between the four countries, the nature and size of the sample did not allow us to draw conclusions at this stage. While we did achieve sampling adequacy through coding saturation across countries, we did not secure this within countries, particularly in Brazil. The nature of the sample, the desire to minimize research burden, ethics, and complex cultural and social challenges within each country meant that we aimed to achieve saturation across our sample, but not within each group. Future research should aim at a better understanding of the specific implementation requirements of the established policy and service principles in different LMIC contexts (e.g. urban vs rural settings), and vulnerable groups (e.g. children in disadvantaged communities, informal settlements, orphanages, or living on the streets). This evidence should inform policy, service planning, and psychosocial interventions, which would require feasibility and substantive evaluation.

\section{Conclusion}

This study involved stakeholders from a range of disciplines in four LMIC, and established their perspectives of meeting the mental health needs of vulnerable children in disadvantaged communities. The findings highlight the importance of joined up policy, which should be reflected on the ground through interdisciplinary working and capacity-building. The active involvement of communities and service users is paramount in maximizing and sustaining the impact of evolving interventions and services.

Acknowledgements

We are grateful to all participants for their involvement. We also thank the four host organizations for the generous facilitation of the study. 


\section{Funding}

This study was supported by a Global Challenges Research Fund, Global Impact Acceleration Award.

Availability of data and materials

The dataset analysed during the current study is available from the corresponding author on reasonable request.

\section{Declarations}

\section{Competing interests}

The authors declare that they have no competing interests.

\section{Author details}

${ }^{1}$ University of Leicester, Leicester, UK. ${ }^{2}$ Necmettin Erbakan University, Konya, Turkey. ${ }^{3}$ University of Johannesburg, Johannesburg, South Africa.

Received: 6 April 2020 Accepted: 3 August 2021

Published online: 12 August 2021

\section{References}

Academy of Medical Sciences. (2018). Challenges and priorities for global mental health in the SDG era. Academy of Medical Sciences.

Adams, N., Daniels, A., \& Compagni, A. (2009). International pathways to mental health transformation. International Journal of Mental Health, 38, 30-45.

Ali, S., Yildirim, M., Hussain, S., \& Vostanis, P. (2020). Self-reported mental health problems and post traumatic stress growth among children in Pakistan care homes. Asia Pacific Journal of Social Work and Development, 30, 62-76.

Arora, P., Connors, E., George, M., Lyon, A., Wok, C., \& Weist, M. (2016). Assessing evidence-based assessment in school mental health. Clinical Child and Family Psychology Review, 19, 271-284.

Bawah, A., Awoonor-Williams, J., Asuming, P., Jackson, E., Boyer, C., Kanmiki, E., Achana, S., Akazili, J., \& Phillips, J. (2019). The child survival impact of the Ghana Essential Health Interventions Program: A health systems strengthening plausibility trial in Northern Ghana. PLoS One. https://doi.org/10.1371/journal.pone.0218025

Bele, S. D., Bodhare, T. N., Valsangkar, S., \& Saraf, A. (2013). An epidemiological study of emotional and behavioral disorders among children in an urban slum. Psychology, Health and Medicine, 18, 223-232.

Bloom, D., Cafiero, E., Jané-Llopis, E., Abrahams-Gessel, S., Bloom, L., Fathima, S., Feigl, A., Gaziano, T., Mowafi, M., Pandya, A., Prettner, K., Rosenberg, L., Seligman, B., Stein, A., \& Weinstein, C. (2011). The global economic burden of non-communicable diseases. World Economic Forum.

Boylan, K. (2018). Measuring children's mental health outcomes from a service perspective. Journal of Canadian Academy of Child and Adolescent Psychiatry, 27, 81. Retrieved from https://www.ncbi.nlm.nih.gov/pmc/articles/PMC5896519.

Braun, V., \& Clarke, V. (2006). Using thematic analysis in psychology. Qualitative Research Psychology, 3, 77-101.

Braun, V., \& Clarke, V. (2019). Reflecting on reflexive thematic analysis. Qualitative Research in Sport, Exercise and Health, 11 , 589-597.

Bronfenbrenner, U. (1979). The ecology of human development. Harvard University Press.

Clarke, V., \& Braun, V. (2018). Using thematic analysis in counselling and psychotherapy research: A critical reflection. Counselling and Psychotherapy Research, 18, 107-110.

Cooper, M., Evans, Y., \& Pubis, J. (2016). Interagency collaboration in children and young people's mental health: A systematic review of outcomes, facilitating factors and inhibiting factors. Child: Care. Health and Development, 42, 325-342.

Dalgaard, N., Todd, B., Daniel, S., \& Montgomery, E. (2016). The transmission of trauma in refugee families: Associations between intra-family trauma communication style, children's attachment security and psychosocial adjustment. Attachment and Human Development, 18, 69-89.

De Los Rayes, A., Augenstein, T., Wang, M., Thomas, S., Drabick, D., Bergers, D., \& Rabinowitz, J. (2015). The validity of the multi-informant approach to assessing child and adolescent mental health. Psychological Bulletin, 141, 858-900.

Economic and Social Research Council (2019). What is impact? Swindon: ESRC. Retrieved from https://esrc.ukri.org/resea rch/impact-toolkit/what-is-impact/pdf. Accessed April 2021

Eruyar, S., Huemer, J., \& Vostanis, P. (2018). How should refugee services respond to the refugee crisis? Child and Adolescent Mental Health, 23, 303-312.

Frankish, H., Boyce, N., \& Horton, R. (2018). Mental health for all: A global goal. Lancet, 392, 1493-1494.

Garcia-Carrion, R., Villarejo-Carballido, B., \& Villardon-Gallego, L. (2019). Children and adolescents mental health: A systematic review of interaction-based interventions in schools and communities. Frontiers in Psychology, 10, 918. https:// doi.org/10.3389/fpsyg.2019.00918

Getanda, E., O'Reilly, M., \& Vostanis, P. (2017). Exploring the challenges of meeting child mental health needs through community engagement in Kenya. Child and Adolescent Mental Health, 22, 201-208.

Green, H., Mc Ginnity, A., Melzer, H., Ford, T., \& Goodman, R. (2005). Mental health of children in Great Britain, 2004. Palgrave macmillan.

He, A., Lim, C., Lecklinter, G., Olson, A., \& Traube, D. (2015). Interagency collaboration and identifying mental health needs in child welfare: Findings from Los Angeles County. Children and Youth Services Review, 53, 39-43.

Hennink, M., Kaiser, B., \& Weber, M. (2017). Code saturation versus meaning saturation: How many interviews are enough? Qualitative Health Research, 27, 591-608.

Hussain, S., Bankart, J., \& Vostanis, P. (2012). Social and educational risk factors for child mental health problems in Karachi, Pakistan. International Journal of Mental Health and Culture, 5, 1-14. 
Institute of Medicine. (2015). Measuring the impact of interprofessional education on collaborative practice and patient outcomes. National Academies Press.

Inter-Agency Standing Committee. (2007). Mental health and psychosocial support in humanitarian emergencies. IASC. Jalal, E., O'Reilly, M., Bhakta, T., \& Vostanis, P. (2019). Barriers to implementing learning from child protection training in Saudi Arabia. International Social Work, 62, 1493-1506.

Jordans, M., Tol, W., \& Komproe, I. (2011). Mental health interventions for children in adversity: Pilot-testing a research strategy for treatment selection in low-income setting. Social Science and Medicine, 73, 456-466.

Kontis, V., Cobb, L., Mathers, C., Frieden, T., Ezzati, M., \& Danai, G. (2019). Three public health interventions could save 94 million lives in 25 years. Circulation, 140, 715-725.

Kumar, M., Huang, K. Y., Othieno, C., Wamalwa, D., Hoagwood, K., Unutzer, J., Saxena, S., Petersen, I., Njuguna, S., Amugune, B., Gachuno, O., Ssewamala, F., \& Mc Kay, M. (2020). Implementing combined WHO mhGAP and group interpersonal psychotherapy to address depression and mental health needs of pregnant adolescents in Kenyan primary health care settings (INSPIRE): A study protocol for pilot feasibility trial of the integrated intervention in LMIC settings. Pilot and Feasibility Studies, 6, 136. https://doi.org/10.1186/s40814-020-00652-8

Lavik, K., Veseth, M., Froysa, H., Binder, P. E., \& Moltu, C. (2018). What are "good outcomes" for adolescents in public mental health settings? International Journal of Mental Health Systems, 12, 3. https://doi.org/10.1186/s13033-018-0183-5

Maslow, A. H. (1943). A theory of human motivation. Psychological Review, 50, 370-396.

Millard, C., \& Wessely, S. (2014). Parity of esteem between physical and mental health. British Medical Journal, 349, g6821. https://doi.org/10.1136/bmj.g6821

Moller, N., Burgess, V., \& Jogiyat, Z. (2016). Barriers to counselling experienced by British South Asian women. Counselling and Psychotherapy Research, 16, 201-210.

Morton, J., \& O'Reilly, M. (2019). Mental health, big data and research ethics: Parity of esteem in mental health research from a UK perspective. Clinical Ethics. https://doi.org/10.1177/2F1477750919876243

Myburgh, C., Moolla, A., \& Poggenpoel, M. (2015). The lived experiences of children living on the streets of Hillbrow. Curationis, 1, 1-8.

Nianogo, R., \& Arah, O. (2018). Impact of public health interventions on obesity and Type 2 diabetes prevention: A simulation study. American Journal of Preventive Medicine, 55, 795-800.

Nilsen, P. (2015). Making sense of implementation theories, models and frameworks. Implementation Science, 10, 53. https://doi.org/10.1186/s13012-015-0242-0

O'Connor, C. A., Dyson, J., Cowdell, F., \& Watson, P. (2017). Do universal school-based mental health promotion programmes improve the mental health of young people? Journal of Clinical Nursing, 27, 3-4. https://doi.org/10.1111/ jocn. 14078

Pandian, R., \& Lakshmana, G. (2017). Risk and resilience factors for substance use among street adolescents. Asian Social Work and Policy Review, 11(3), 216-233.

Patel, V., \& Rahman, A. (2015). An agenda for child mental health. Child and Adolescent Mental Health, 20, 3-4.

Patel, V., Saxena, S., Lund, C., Thornicroft, G., Baingana, F., Bolton, P., Chisholm, D., Collins, P. Y., Cooper, J. L., Eaton, J., Herrman, H., Herzallah, M. M., Huang, Y., Jordans, M. J. D., Kleinman, A., Medina-Mora, M. E., Morgan, E., Niaz, U., Omigbodun, O., ... UnÜtzer, J. (2018). The Lancet Commission on global mental health and sustainable development. Lancet, 392, 1553-1598.

Pawson, R., \& Tilley, N. (1997). Introduction to scientific realist evaluation. In E. Chelimsky \& W. Shadish (Eds.), Evaluation for the 21st Century (pp. 405-418). Sage.

Pedersen, G., Zajkowska, Z., Keiling, C., Gautam, K., Mondelli, V., Fisher, H., Swartz, J., Adewya, A., Karmacharya, R., \& Kohrt, B. (2019). Protocol for a systematic review of the development of depression among adolescents and young adults: Psychological, biological, and contextual perspectives around the world. Systematic Reviews, 8, 179. https://doi.org/ 10.1186/s13643-019-1104-7

Purgato, M., Gross, A., Jordans, M., de Jong, J., Barbui, C., \& Tol, W. (2014). Psychosocial interventions for children exposed to traumatic events in low- and middle-income countries. Systematic Reviews, 3, 34. https://doi.org/10.1186/ 2046-4053-3-34

Reardon, T., Harvey, K., Baranowska, M., O'Brien, D., Smith, L., \& Creswell, C. (2017). What do parents perceive are the barriers and facilitators to accessing psychological treatment for mental health problems in children and adolescents? A systematic review of qualitative and quantitative studies. European Child and Adolescent Psychiatry, 26, 623-647.

Reza, M., \& Bromfield, N. F. (2019). Human rights violations against street children working in the informal economy in Bangladesh. Journal of Human Rights and Social Work, 4, 1-12.

Schnyder, N., Panczak, R., Groth, N., \& Schultze-Lutter, F. (2017). Association between mental health-related stigma and active help-seeking: Systematic review and meta-analysis. The British Journal of Psychiatry, 210(4), 261-268.

Skokauskas, N., Fung, D., Flaherty, L., Klitzing, K. V., Pūras, D., Servili, C., Dua, T., Falissard, B., Vostanis, P., Moyano, M. B., Feldman, I., Clark, C., Boričević, V., Patton, G., Leventhal, B., \& Guerrero, A. (2019). Shaping the future for child and adolescent psychiatry. Child and Adolescent Psychiatry. https://doi.org/10.1186/s13034-019-0279-y

Tamburrino, I., Getanda, E., O'Reilly, M., \& Vostanis, P. (2020). "Everybody's responsibility”: Conceptualisation of youth mental health in Kenya. Journal of Child Health Care, 24, 5-18.

United Nations. (2014). Social inclusion of youth with mental health conditions. UN.

UNHCR. (2013). Operational guidance: Mental health and psychosocial support programming for refugee operations. UNHCR.

United Nations Human Settlement Programme (2017). Annual Progress Report. Nairobi: UN Habitat. Retrieved from https://unhabitat.org/wp-content/uploads/2018/06/Annual-Progress-Report-2017_-16-APR.pdf.

Vostanis, P. (2017). Global child mental health: Emerging challenges and opportunities. Child and Adolescent Mental Health, 22, 177-178.

Vostanis, P. (2019). World Awareness for Children in Trauma: Capacity-building activities of a psychosocial program. International Journal of Mental Health, 48, 323-329.

Vostanis, P., Eruyar, S., Smit, E., \& O'Reilly, M. (2019). Development of child psychosocial framework in Kenya, Turkey and Brazil. Journal of Children's Services, 14, 303-316. 
Vostanis, P., Maltby, J., Duncan, C., \& O'Reilly, M. (2018). Stakeholder perspectives on children's psychosocial needs in six low- and middle-income countries. Children and Society, 32, 457-469.

Vostanis, P., Martin, P., Davies, R., De Francesco, D., Jones, M., Sweeting, R., Ritchie, B., Allen, P., \& Wolpert, M. (2015). Development of a framework for prospective payment for child mental health services. Journal of Health Services Research and Policy, 20, 202-209.

Wolpert, M., Vostanis, P., Martin, K., Munk, S., Norman, R., Fonagy, P., \& Feltham, A. (2017). High integrity child mental health: Focusing on the person, not the problem. British Medical Journal, 22, 138-147.

World Health Organization. (2010). Framework for Action on Interprofessional Education and Collaborative Practice. WHO World Health Organization. (2014). Integrating the response to mental disorders and other chronic diseases in healthcare systems. WHO.

World Health Organization. (2016). Mental health: Strengthening response. WHO.

\section{Publisher's Note}

Springer Nature remains neutral with regard to jurisdictional claims in published maps and institutional affiliations.

\section{Submit your manuscript to a SpringerOpen ${ }^{\circ}$} journal and benefit from:

- Convenient online submission

- Rigorous peer review

Open access: articles freely available online

- High visibility within the field

Retaining the copyright to your article

Submit your next manuscript at $\gg$ springeropen.com 